MS31-02

\section{The role of supramolecular interactions in the formation of isostructural and polymorphic structures}

Petra Bombicz ${ }^{1}$

1. Research Centre for Natural Sciences, Hungarian Academy of Sciences, Budapest, Hungary

email: bombicz.petra@ttk.mta.hu

The non-covalent interactions have an influence on seeding and crystal growth, recognition processes, the packing arrangement and the molecular conformation, as well as they affect the stability of the crystal and the biological activity of the compounds.

Both polymorphism and isostructurality are strongly related to intermolecular interactions and crystal symmetries [1]. Structures with similar chemical content may be isostructural. The difference can be in the substitution of the molecule(s) or in the guest molecule in inclusion systems. But how far can a crystal structure tolerate small molecular changes? The molecular conformation of flexible molecules may adjust to the altering supramolecular features in the crystal lattice accompanying chemical changes. It is a subject of debate how far two similar crystal structures can be different to consider them as isostructural. Nevertheless, a higher crystal symmetry can be sacrificed to achieve a better supramolecular arrangement resulting in a lower crystal symmetry, that is, resulting in the introduction of pseudosymmetry and, with that, Z'> 1 crystal packing.

The identity of the supramolecular interactions does not ensure isostructurality. On the other hand the occurrence of common crystal packing patterns amongst polymorphs is frequent.

Partial isostructurality may explain the macroscopic behaviour of compounds revealing molecular structure-macroscopical property relationships. Epitaxial crystal growth of compounds becomes possible at a surface where two-dimensional isostructurality is realized, enabling the further growth of the supramolecular pattern in that direction.

A special aspect is the intergrowth of domain arrangements within crystals including varying supramolecular pattern and/or symmetries at the domains' surface having a variable domain proportion resulting in crystals with structurally continuously altering polymorphs and thus in crystals which possess continuously varying physico-chemical properties.

Recognition of the structural features, the supramolecular and symmetry aspects of isostructurality and polymorphism contributes to the proficiency of synthon engineering, the manipulation of the intermolecular interactions, and which makes it possible to engineer crystals.

\section{Acknowledgement:}

The support of the National Research, Development and Innovation Office - NKFIH through OTKA K124544 is gratefully acknowledged.

References:

[1] Bombicz, P. (2017) Crystallography Reviews 23(2),

118-151.Keywords: supramolecular interactions, polymorphism, isostructurality

Keywords: supramolecular interactions, polymorphism, isostructurality
MS31-03

\section{Cocrystallisation enhanced optical properties of organic materials - quantum crystallography meets crystal engineering}

Marlena Gryl ${ }^{1}$, Tomasz Seidler ${ }^{1}$, Anna Krawczuk ${ }^{1}$, Katarzyna Ostrowska ${ }^{1}$, Katarzyna Stadnicka ${ }^{1}$

1. Faculty of Chemistry, Jagiellonian University, Kraków, Poland email: gryl@chemia.uj.edu.pl

During the last decade optical materials have experienced a rebirth of scientific interest. This phenomenon can be explained by the rapidly developing fields of optoelectronics and overall minimization in telecommunication devices. The need for more efficient, stable, and low price materials promotes multicomponent and multifunctional systems over those with single component and single functionality. Additionally usage of more than one building block in a single material enables both steering of the crystal symmetry towards a specific property and at the same time gives means for enhancing the desired physical and/or chemical effect. Co-crystallization is a widely used technique in materials science to obtain materials with modified physiochemical properties or enforce particular crystal symmetry.

Here we present our approach towards crystal engineering of optical materials ${ }^{1}$. Birefringence, second harmonic generation, absorption and/or fluorescence can be modified through controlled cocrystallisation of suitable building blocks: one to guarantee a substantial property at the molecular level (suitable chromophore) and the second one selected for controlling crystal symmetry. Combination of quantum crystallography techniques aimed towards analysis of intermolecular interactions $\left(\mathrm{QTAIM}^{2}, \mathrm{NCI}\right.$ index ${ }^{3}$ ) were used alongside with calculations of molecular and bulk properties of the studied systems. Theoretical methodology was supported by measurements of the selected optical properties of the obtained materials.

References:

[1] Gryl, M. (2015). Acta Cryst. B71, 392-405.

[2] Bader, R. F. W. (3003), Atoms in molecules, A Quantum Theory. New York: Oxford University Press.

[3] Contreras-Garciá, et al., (2011). J. Chem. Theory Comput., 7, 625-632.

Keywords: cocrystallisation, optical materials, crystal engineering 